\title{
What-To-Taste: A Food Recommendation System
}

\author{
Kashish Ahuja, Mukul Goel, Sunil Sikka, Priyanka Makkar
}

\begin{abstract}
The Food recommendation system software is relatively new in this era, with the recommendation system that focuses on the user preferences posited. Generally we get confused about what to eat or try next, this problem is solved by our project, which recommends food according to customer's experience, customer's ratings on cuisine and customer's taste.

"What-To-Taste" is a food recommendation system which can be used by the food chain industry to keep recommending their customers about the cuisine, basically whatever they have in their menu, based on their customer tastes, interests and order history. Each human has a different taste that can be verified from recommendation data at every new place and the best cuisine served is location-dependent by rating through the customer.

The Food recommendation system recommends by Food profiling, User profiling, and recommending the specific food item as per the last feedback submitted by the users.
\end{abstract}

KEYWORDS- Content Based approach, Information Retrieval, Collaborative Based Approach.

\section{INTRODUCTION}

Food is our part of living tradition and culture [1]. It means that eating has an emotional part for many people. People make decisions every day, "What should I eat? "The way a person or group gets confused with what, how, when and, how much to eat, if we consider the present era then there is a big concern among youth regarding several food-related information where intake amount and safety of food are the two main concerns that almost everyone face nowadays. And these all have explosively assembled through food chain resources.

\section{Manuscript received May 2, 2020}

Kashish Ahuja, Department of Computer Science, AmityUniversity,Gurgaon,Haryana,India(e-mail:ahujakas hish86@gamil.com).

Mukul Goel, Department of Computer Science, Amity University, Gurgaon, Haryana, India

Sunil Sikka, Department of Computer Science, Amity University, Gurgaon ,Haryana,

Priyanka Makkar, Department of Computer Science, Amity University, Gurgaon, Haryana, India
The resources have become an open store of new nourishment data, going from exceptionally broad data to new logical realities identified with explicit fixings, or nourishments drawn from the way of life, scholastic diaries, and government and research foundations. It opens another entryway for individuals to pine for nourishment. The current paper presents a food recommendation system with the aim to induce connections between different nations' cooking styles, concentrating on nourishment alternatives, specifically, in light of taste, choice exposure, and experience. Specifically, this system used information about what to eat appearing in data in our food chain industry[9] compared to other countries we are also keen on inducing the elements driving any likenesses in nourishment proposal rating utilization that may develop between nations. The proposed system is a basically a content-based recommendation system with a bit touch of collaborative systems. For the items related to search the Recommendation Problems are treated by the approaches which are content based. Given rated food, the algorithm performs a function to find another equivalent item. In the system, nourishment are characterized by their sustenance highlight and spoken to by vectors. However, this is the easiest idea driving substance-based proposal frameworks. Following are the advantages of proposed food recommendation system

a) The benefit to the customers:

The food recommendation system provides the exact and the straight away recommendations about the food item and shows its rating given by customers [2]. The proposed system will allow customers to ponder about what to eat. This system also provides recommendation of the top restaurants on the basis of votes and ratings.

b) Benefit to the food chain industry

Only food domains are functioned and controlled by the system especially the domains that have registered or login in the software. This is implemented by using user feedback.

c) Cost efficient and user friendly

The system is cost efficient as well as user-friendly.

The software used to develop the system includes Sublime Text Editor, Localhost Sm erver, Python 3, Flask, Machine Learning Packages (Scikit Learn, Pandas, Numpy, Bs4)[13], Web Browser. It requires a very less cost to complete its design. 
Developed Food recommendation systems helps to understand the techniques of recommending food item by including various factors like nutrients value, user's preferences and keywords and tags. Each human has a different taste that can be verified from recommendation data at every new location and the best cuisine served is location-dependent by rating through the customer. The current model serves to the taste of users by recommending food items based on other users, categories, place and item profiling. The feature of our project is the simplicity and the fact that you can get recommendations accordingly for food as well as restaurants and at the same time you can order also. Rest of the paper is divided into three sections. Section II discusses the various approaches available to develop a recommendation system. Section III presents the detail of proposed food recommendation system.

\section{RECOMMENDATION TECHNIQUES}

The use of efficient and accurate recommendation techniques is extremely important for a system which will provide good and useful recommendation to its individual users. This explains the importance of understanding the features and potentials of various recommendation techniques. There are many recommendation techniques available within the literature. a number of the commonly used techniques are as follows.

\section{A. Simple Recommendation}

This technique is predicated on the weighted rating. The weighted rating of two or more parameters is employed to calculate an efficient score on the idea of which we sort items of the idea of recommendations.

\section{B. Content Based Recommendation[18]}

Content-based technique may be a domain-dependent algorithm and it emphasizes more on the analysis of the attributes of things so as to get predictions. When documents like sites, publications and news are to be recommended, content-based filtering technique is that the most successful. In content-based filtering technique, recommendation is formed supported the user profiles using features extracted from the content of the things the user has evaluated within the past. Items that are mostly associated with the positively rated items are recommended to the user.

Content Based Filtering uses differing types of models to seek out similarity between documents so as to get meaningful recommendations. Pros and Cons of content-based filtering techniques Content based filtering techniques overcome the challenges of Collaborative filtering. They need the power to recommend new items albeit there are not any ratings provided by users. So albeit the database doesn't contain user preferences, recommendation accuracy isn't affected. Also, if the user preferences change, it's the capacity to regulate its recommendations during a short span of your time.

They will manage situations where different users don't share an equivalent items, but only identical items consistent with their intrinsic features. Content based filtering techniques are hooked in to items' metadata. That is, they require rich description of things and really well organized user profile before recommendation are often made to users. this is often called limited content analysis. So, the effectiveness of Content Based Filtering depends on the supply of descriptive data. Users are restricted to getting recommendations almost like items already defined in their profiles.

\section{Collaborative Recommendation[12,18]}

Collaborative filtering may be a domain-independent prediction technique for content that can't easily and adequately be described by metadata like movies and music. Collaborative filtering technique works by building a database (user-item matrix) of preferences for items by users. It then matches users with relevant interest and preferences by calculating similarities between their profiles to form recommendations. Such users build a gaggle called neighborhood. An user gets recommendations to those items that he has not rated before but that were already positively rated by users in his neighborhood. Recommendations that are produced by Collaborative filtering are often of either prediction or recommendation

\section{SYSTEM DESCRIPTION}

Recommendation programming is written in Python3 programming language [7]. It acts as a conversion tool and converts the used programming language into bytecode and, directly bytecode is loaded in system memory.

It is basic, natural, simple to utilize, simple to control numerous clients simultaneously, and has great machine-learning algorithms, support for web services (Flask) [13], XML \& database systems, etc. software is developed on windows and the CSV file is used as the dataset.

WHAT-TO-TASTE is a web application recommendation system that tracks the user's food intake by his interests.

We use latest machine learning techniques to recognize users, making instant ratings and similar food items estimates from the user's meals.

The system also suggests restaurants based on the user's choices, and is capable of showing places nearby that serve those dishes.

Restaurants can use this system to offer meals, dishes to their customers based on their interests and hence can please user with high quality services 


\section{A. System Design}
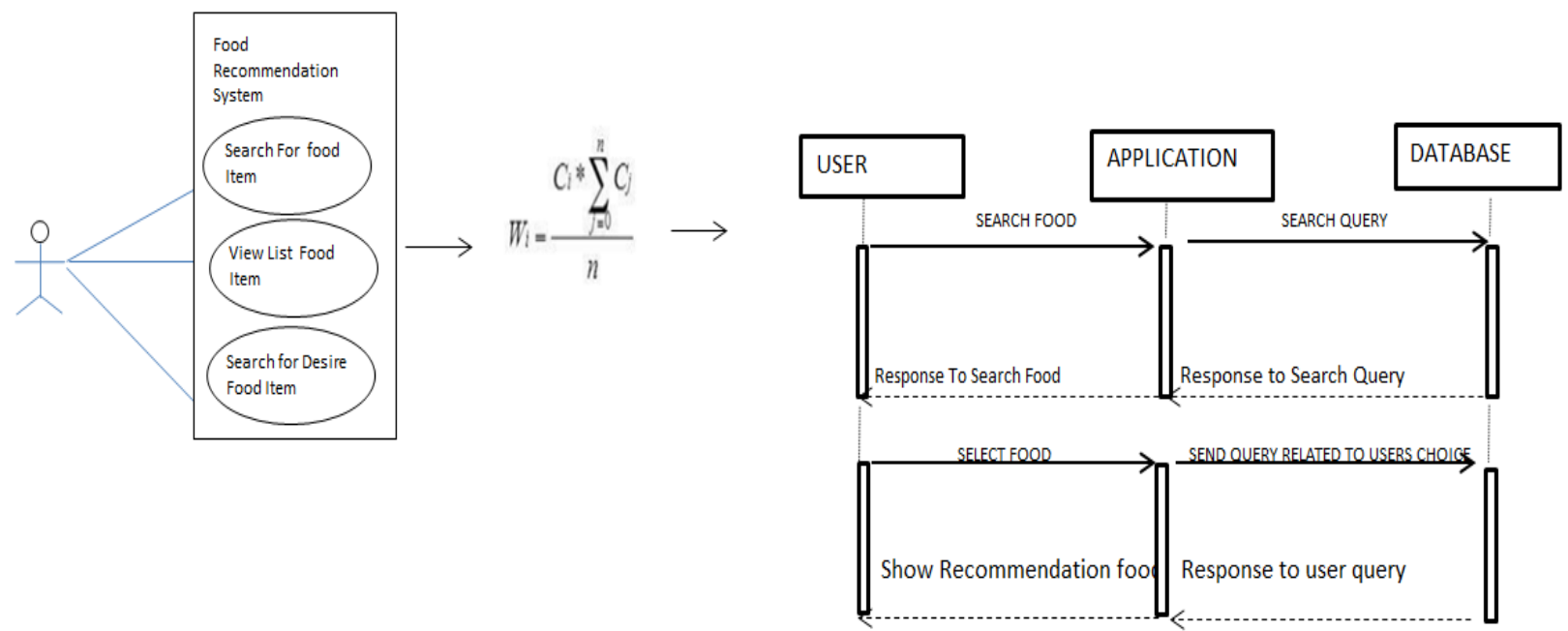

Fig. 1: System Functionality

The block diagrams of the food system and receiver side were explained above

\section{B. Methodology}

Information is available to all users through information systems during hours of operation to show them the

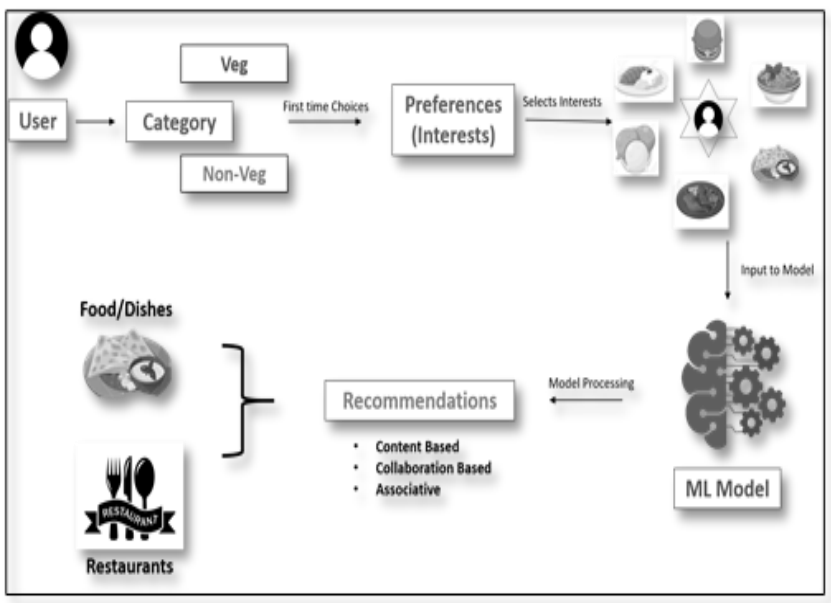

Fig. 2: Flowchart

required recommendations. The content-based approach [3] treats the recommendation problem as a search from the collected data. It is portable as it runs anywhere. The software allows for the addition or deletion of Users, Dishes, and restaurants while incorporating new semesters. The framework is designed in such a way that foods are defined by their significant qualities and are spoke to by vectors and this is why feature weights are important in these vectors and it always seeks to search out the best match among the food profile and the user profile. Check out some of the most preferred.

\section{Working}

The above diagram explains the working of our food recommendation system. A user selects his top food preferences and food category at the beginning, in order to get top recommendations based on his preferences. Based on this user profiling and item profiling, the recommendation system will work to show him the recommendations accordingly.[15]

Various filters and choices are given to user in order to get recommendations:[17]

a) Users can search for a particular food item like burger, and get recommendations for top burgers.

b) Users can get recommendations for a particular food category like fast foods, desserts, Indian etc.

c) Users can get recommendations based on other users that is collaborative approach.

d) Users can also get recommendations for top restaurants in the area.

e) User can even order recommended food item from the Zomato's website.

\section{RESULTS}

In WHAT-TO-TASTE food recommendation System, there is no need for an individual for waiting long, and waste their time in thinking about what to eat, Instead we use State-of-the-art techniques in machine learning to recognize users, making instant ratings and similar food items estimates from the user's meals, item profiling and hence make instant food recommendations according to user needs. The system also suggests restaurants based on the user's choices, and is capable of showing places nearby that serve those dishes. The project overall facilitates the user taste needs, and quench his insatiable thirst of searching fresh food items and cuisines by using recommendation techniques. 


\section{CONCLUSION AND FUTURE SCOPE}

The Project "WHAT-TO-TASTE" has a tremendous extension in future. The undertaking can be actualized in eateries in future. The refreshing should be possible in venture in future according to the necessities, Due to its element of adaptability concerning extension. With the proposed arrangement of WHAT-TO-TASTE, the task is prepared and completely useful the client can undoubtedly get suggestions for their food advantages.

The Future Scopes of the project are as follows.

- Adding the Nutritional factor in the process of recommendations. The project can then, recommend food considering its nutritional value as well to suggest some healthy recommendations.

- Suggesting better personalized recommendations through analyzing user data, history and cookies.

- Converting the Web Application into an on the go android application, so the system come in handy and is easily accessible for daily life use.

\section{REFERENCES}

[1]Sibal, Vatika.. Food: Identity of Culture and Religion. July-Aug, 2018 Volume 6, 10908-10915)

[2]D.S.Gaikwad,Anup.V.Deshpande,A Recommendation System

Food Maharashtra,2017,p-ISSN: 2395-0072

[3] Content-based filtering algorithm http://recommender-systems.org/content-based-filterin g/

[4]Faisal Rehman,Osman Khalid(2017),Diet-Right: A Smart Food Recommendation System,DOI: 10.3837/tiis.2017.06.006

[5]Trattner, Christoph \& Elsweiler, David. (2017). Food Recommender Systems: Important Contributions, Challenges and Future Research Directions.

[6]Min, W., Jiang, S., \& Jain, R. C. (2019). Food Recommendation: Framework, Existing Solutions and Challenges. IEEE Transactions on Multimedia,p-ISSN: 1520-9210, DOI: 10.1109/TMM.2019.2958761

[7]Varga E. Recommender Systems. In: Practical Data Science with Python 3. Apress, Berkeley, CA,2019, pISBN 978-1-4842-4858-4.

[8]https://towardsdatascience.com/introduction-to-recom mender-systems-6c66cf15ada

[9] Carroll County Foods "food chain industry https://industrytoday.com/food-chain

[10]Shuvayan Das (11/08/2015) Beginner Guide to learn Content Based Recommender Engines. Analytics Vidhya.

[11]Thom Hopmans (11/02/2016) A recommendation system for blogs: Content-based similarity (part 2). The Marketing Technologist.

https://www.themarketingtechnologist.co/a-recommen dation-system-for-blogs-content-based-similarity-part2/

[12]Kunal Deshmukh (16/03/2018) Collaborative Filtering in Recommendation Systems. Medium.com https://medium.com/kunalrdeshmukh/collaborative-filt ering-in-recommendation-systems-2fa49be8f518
[13]Guillaume Ferry. Python Machine Learning Prediction with a Flask Rest API. Toptal.com.

https://www.toptal.com/python/python-machine-learni ng-flask-example

[14]Breakfast. Better Health Channel. https://www.betterhealth.vic.gov.au/health/healthylivi ng/breakfast

[15] Recommender System Wikipedia. https://en.wikipedia.org/wiki/Recommender_system

[16]Tavish Srivastava (07/04/2015) Information Retrieval System explained in simple terms. Analytics Vidhya. https://www.analyticsvidhya.com/blog/2015/04/inform ation-retrieval-system-explained/

[17] Faith Gedikli (29/03/2013) 'Recommendation System and the social web' Springer https://books.google.co.in/books?id=YWVEAAAAQB AJ\&pg=PA23\&lpg=PA23\&dq= false

[18]F.O.Isinkaye, Y.O.Folajimi,B.A.Ojokoh,Recommendat ion systems:Principles,methods and evaluation,Egyptian Informatics Journal,2015,pages 261-273 